\title{
论 文 祝贺王自强院士80华诞专辑
}

\section{基于内能分解的力-热-化多物理场堣合系统连续 介质力学}

\author{
郑家宏 ${ }^{1}$, 蒋聪盈 $^{1}$, 仲政 ${ }^{2 *}$ \\ 1. 同济大学航空航天与力学学院, 上海 200092; \\ 2. 哈尔滨工业大学(深圳)理学院, 深圳 518055 \\ * E-mail: zhongzheng@hit.edu.cn
}

收稿日期: 2019-04-03; 接受日期: 2019-06-17; 网络版发表日期: 2019-10-08

国家重点研发计划(编号: 2018YFB1502600)和国家自然科学基金(批准号: 11772106, 11572227)资助项目

摘要 本文将内能分解为可逆自由内能和不可逆耗散能两部分, 基于连续介质热力学获得了考虑传热、传质、 化学反应和宏观形变的开放系统守恒律, 以及相应的力-热-化多场耦合本构关系和演化方程的表达式, 从而建立 了基于内能分解的力-热-化多场耦合理论框架以及等效积分弱形式，最后给出了一个典型的力-化耦合问题数值 算例.

关键词连续介质热力学, 多物理场耦合, 自由能, 化学反应

\section{1 引言}

随着对先进功能材料 ${ }^{[1,2]}$ 、能量存储与转化材 料 ${ }^{[3,4]}$ 、天然多孔介质 ${ }^{[5 \sim 7]}$ 、天然纤维增强复合材料 ${ }^{[8,9]}$ 和生物组织 ${ }^{[10,11]}$ 等研究的不断深入，多场耦合问题日 益得到重视. 早期的多场耦合理论多关注应力和传质 之间的关系. 达西定律唯象地研究了流速与应力梯度 的关系 ${ }^{[7]} ; \mathrm{Gibbs}^{[12]}$ 研究了组分浓度与压力之间的关 系; $\mathrm{Biot}^{[13]}$ 在上述工作的基础上，发展了多孔弹性力 学, 系统地研究了多孔介质中的扩散和流动问题. 后 继的研究更一般地从宏观场论和混合物理论出发探索 力场、热场、扩散场等之间的耦合现象. De Groot和 Mazur $^{[14]}$ 建立了封闭系统的多物理场非平衡热力学理
论; Chester等人 ${ }^{[2,15]}$ 、Hong 等人 ${ }^{[16]}$ 和Yang等人 ${ }^{[17]}$ 针对 不同的应用背景提出了多物理场理论. 近期多场耦合 研究的焦点之一是力学响应与化学反应之间的耦 合 ${ }^{[18 ~ 21]}$. Zhang和Zhong ${ }^{[22]}$ 提出的理论框架, 区分了耦 合效应中化学反应与扩散所引起的不同贡献. 在多场 耦合应用的计算模拟方面，Yang等人 ${ }^{[17]}$ 建立了多场耦 合变分原理及相应的有限元格式. Yu和Shen ${ }^{[18]}$, Chen 等人 ${ }^{[20]}$ 基于能量守恒提出的物理变分原理, 为多场耦 合的计算模拟提供了理论基础. 但是普适的多场耦合 力学数值计算方法仍有待进一步探究.

本文根据热力学系统的可逆与不可逆因素, 对内 能进行分解, 构建了一套基于内能分解的力-热-化多 场耦合理论框架，使得热场的平衡方程也可与其他物

引用格式: 郑家宏, 蒋聪盈, 仲政. 基于内能分解的力-热-化多物理场耦合系统连续介质力学. 中国科学: 技术科学, 2019, 49: 1168-1176 Zheng J H, Jiang C Y, Zhong Z. Continuum mechanics for thermo-chemo-mechanical coupling system based on decomposition of internal energy (in Chinese). Sci Sin Tech, 2019, 49: 1168-1176, doi: 10.1360/SST-2019-0053 
理场进行形式上的类比, 并且在热力学上与已有的理 论是一致的. 这样一套新的多场耦合理论框架, 具有 简洁的形式和清晰的物理意义, 为多场耦合理论分析 提供了一种新的途径.

\section{2 力-热-化多场耦合开放系统的守恒律}

考虑一个由化学活性介质组成的宏观均匀物体 $\mathrm{B}$, 其边界为 $\partial \mathrm{B}$. 在 $\mathrm{B}$ 中选取一个任意的体积单元 $\Omega$, 并记 $\mathbf{n}$ 为其边界 $\partial \Omega$ 的单位外法向矢量. 对于这样的开放系 统, 热力学第一定律可以表示为

$\frac{\mathrm{d} U}{\mathrm{~d} t}=\frac{\mathrm{d} W}{\mathrm{~d} t}$,

其中, $U=E+K, E$ 为系统内能, $K$ 为系统动能; $W=W_{F}+W_{Q}$ $+W_{\mu}, W_{F}$ 为外界对系统所做的机械功, $W_{Q}$ 为外界直接 输入系统的热量, $W_{\mu}$ 为外界物质输入系统所带来的能 量; $\mathrm{d} / \mathrm{d} t$ 表示物质导数. 上述物理量可以进一步表示为

$$
\begin{aligned}
E= & \int_{\Omega} e \mathrm{~d} V, \\
K= & \sum_{\alpha} \frac{1}{2} \int_{\Omega} \rho_{\alpha} \mathbf{v}_{\alpha} \cdot \mathbf{v}_{\alpha} \mathrm{d} V, \\
\dot{W}_{F}= & \int_{\Omega} \mathbf{f} \cdot \mathbf{v} \mathrm{d} V+\int_{\partial \Omega} \mathbf{T} \cdot \mathbf{v} \mathrm{d} S, \\
\dot{W}_{Q}= & -\int_{\partial \Omega} \mathbf{j}_{q} \cdot \mathbf{n} \mathrm{d} S, \\
\dot{W}_{\mu}= & -\sum_{\alpha} \int_{\partial \Omega} \mu_{\alpha} \mathbf{j}_{\alpha}^{\mathrm{m}} \mathrm{d} S \\
& -\sum_{\alpha} \int_{\partial \Omega} \frac{1}{2} M_{\alpha} \mathbf{j}_{\alpha}^{\mathrm{m}}\left(\mathbf{v}_{\alpha} \cdot \mathbf{v}_{\alpha}\right) \mathrm{d} S,
\end{aligned}
$$

式中, $e$ 为系统的单位体积内能, $\rho_{\alpha}$ 为 $\alpha$ 物质的密度; $\mathbf{u}$ 为 质点质心的位移矢量, $\mathbf{v}=\dot{\mathbf{u}}$ 为质点质心的速度矢量, $\mathbf{v}_{\alpha}$ 为 $\alpha$ 物质的速度矢量; $\mathbf{f}$ 为体积力, $\mathbf{T}=\boldsymbol{\sigma} \cdot \mathbf{n}$ 为面力, $\boldsymbol{\sigma}$ 为 Cauchy应力; $\mathbf{j}_{q}$ 为单位面积热流矢量 $\left(\mathrm{J} / \mathrm{m}^{2}\right), \mathbf{j}_{\alpha}^{\mathrm{m}}$ 是单位 面积 $\alpha$ 物质扩散流矢量 $\left(\mathrm{mol} / \mathrm{m}^{2}\right) ; \mu_{\alpha}$ 为 $\alpha$ 物质的化学势, $M_{\alpha}$ 为 $\alpha$ 物质的摩尔质量, 对 $\alpha$ 物质的摩尔浓度 $\bar{c}_{\alpha}$, 有 $M_{\alpha} \check{c}_{\alpha}=\rho_{\alpha}$. 质点的平均密度 $\rho$ 和质心速度 $\mathbf{v}$ 定义为

$\rho=\sum_{\alpha} \rho_{\alpha}, \rho \mathbf{v}=\sum_{\alpha} \rho_{\alpha} \mathbf{v}_{\alpha}$.

相应地, 物质导数可以表示为

$\frac{\mathrm{d}}{\mathrm{d} t}=\frac{\partial}{\partial t}+\mathbf{v} \cdot \nabla=\dot{\mathrm{c}}$

其中, $\partial / \partial t$ 为局部导数, $\nabla$ 为梯度算子.
在一些连续介质热力学理论中 ${ }^{[14,23]}$, 将动能密度 表示为 $\rho \mathbf{v} \cdot \mathbf{v} / 2$, 这仅适用于各组分速度与质心速度相 差不大的情况 (误差 $\propto\left\|\mathbf{v}-\mathbf{v}_{\alpha}\right\|^{2}$ ). 对于封闭系统, 即与 周围环境没有质量交换、只发生系统内部组分的扩 散, 则式(6)可以省略, 许多混合物理论 ${ }^{[24]}$ 都是基于这 样的假设, 但对于开放系统, 必须考虑式(6).

由 $\Omega$ 的任意性, 得到能量平衡方程式(1)的局域 形式:

$\dot{e}+\sum_{\alpha} \rho_{\alpha} \mathbf{v}_{\alpha} \cdot \dot{\mathbf{v}}_{\alpha}+\frac{1}{2} \sum_{\alpha} \dot{\rho}_{\alpha} \mathbf{v}_{\alpha} \cdot \mathbf{v}_{\alpha}$

$+\left(e+\frac{1}{2} \sum_{\alpha} \rho_{\alpha} \mathbf{v}_{\alpha} \cdot \mathbf{v}_{\alpha}\right) \nabla \cdot \mathbf{v}$

$=\mathbf{f} \cdot \mathbf{v}+\mathbf{v} \cdot \nabla \cdot \boldsymbol{\sigma}+\boldsymbol{\sigma}: \nabla \mathbf{v}$

$-\nabla \cdot \mathbf{j}_{q}-\sum_{\alpha} \nabla \cdot\left(\mu_{\alpha} \mathbf{j}_{\alpha}^{\mathrm{m}}\right)$

$-\sum_{\alpha} \nabla \cdot \frac{M_{\alpha} \mathbf{j}_{\alpha}^{\mathrm{m}}}{2}\left(\mathbf{v}_{\alpha} \cdot \mathbf{v}_{\alpha}\right)$.

根据伽利略相对性原理, 可以进一步证明

$\sum_{\alpha}\left(\dot{\rho}_{\alpha}+\rho_{\alpha} \nabla \cdot \mathbf{v}+\nabla \cdot M_{\alpha} \mathbf{j}_{\alpha}^{\mathrm{m}}\right)=0$,

$\sum_{\alpha} \rho_{\alpha} \dot{\mathbf{v}}_{\alpha}-\nabla \cdot \boldsymbol{\sigma}-\mathbf{f}=0$.

对任意物质 $\alpha$ 都有

$$
\begin{aligned}
\left(\dot{\rho}_{\alpha}+\rho_{\alpha} \nabla \cdot \mathbf{v}\right) / M_{\alpha} & =-\nabla \cdot \mathbf{j}_{\alpha}^{\mathrm{m}}+\dot{r}_{\alpha} \\
& =\dot{c}_{\alpha}+\sum_{\gamma} v_{\alpha}^{(\gamma)} \dot{\ell}^{(\gamma)},
\end{aligned}
$$

式中, $\dot{c}_{\alpha}=-\nabla \cdot \mathbf{j}_{\alpha}^{\mathrm{m}}$, 称为扩散浓度; $r_{\alpha}=\sum_{\gamma} v_{\alpha}^{(\gamma)} \ell^{(\gamma)}$ 为 $\alpha$ 物质的源项, $v_{\alpha}^{(\gamma)}$ 为化学反应 $\gamma$ 中 $\alpha$ 物质的化学计量数, $\ell^{(\gamma)}$ 为化学反应 $\gamma$ 的反应进度. 式(12)对 $\alpha$ 求和即得质量 守恒式(10). 再利用物质密度与摩尔浓度之间的关 系 $M_{\alpha} \bar{c}_{\alpha}=\rho_{\alpha}$, 改写式(12)并积分可得

$$
\begin{aligned}
\frac{\mathrm{d}}{\mathrm{d} t} \int_{\Omega} \check{c}_{\alpha} \mathrm{d} V & =\int_{\Omega}\left(\dot{c}_{\alpha}+\sum_{(\gamma)} v_{\alpha}^{(\gamma)} \dot{\ell}^{(\gamma)}\right) \mathrm{d} V \\
& =-\int_{\partial \Omega} \mathbf{j}_{\alpha}^{\mathrm{m}} \cdot \mathbf{n} \mathrm{d} S+\int_{\Omega} \dot{r}_{\alpha} \mathrm{d} V,
\end{aligned}
$$

或写为局域形式:

$\dot{c}_{\alpha}+\breve{c}_{\alpha} \nabla \cdot \mathbf{v}=\dot{c}_{\alpha}+\sum_{(\gamma)} v_{\alpha}^{(\gamma)} \dot{\ell}^{(\gamma)}=-\nabla \cdot \mathbf{j}_{\alpha}^{\mathrm{m}}+\dot{r}_{\alpha}$.

式(11)和(14)即为开放系统的动量守恒方程和质量守 恒方程.

能量守恒方程(9)因此可以化为

$\dot{e}+e \nabla \cdot \mathbf{v}=\boldsymbol{\sigma}: \nabla \mathbf{v}-\nabla \cdot \mathbf{j}_{q}-\sum_{\alpha} \nabla \cdot\left(\mu_{\alpha} \mathbf{j}_{\alpha}^{\mathrm{m}}\right)$. 


\section{3 内能的分解}

本节将在上述能量守恒方程的基础上，根据物理 含义, 给出本文所提出的内能分解形式.

在小变形假设下可忽略体积变化率(即忽略能量 守恒方程(15)中含速度散度的项), 进而将内能平衡方 程式(15)改写为

$$
\begin{aligned}
\dot{e}= & \boldsymbol{\sigma}: \dot{\boldsymbol{\varepsilon}}^{\mathrm{r}}+\boldsymbol{\sigma}: \dot{\boldsymbol{\varepsilon}}^{\mathrm{i}}-T \nabla \cdot\left(\frac{\mathbf{j}_{q}}{T}\right)-\frac{\mathbf{j}_{q}}{T} \cdot \nabla T \\
& +\sum_{\alpha} \mu_{\alpha} \dot{c}_{\alpha}-\sum_{\alpha} \mathbf{j}_{\alpha}^{\mathrm{m}} \cdot \nabla \mu_{\alpha} .
\end{aligned}
$$

这里利用了 $\dot{c}_{\alpha}=-\nabla \cdot \mathbf{j}_{\alpha}^{\mathrm{m}}$ 及应变率的加法分解, 即

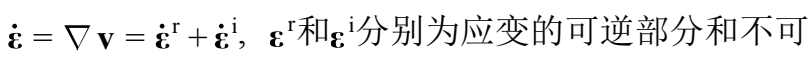
逆部分 ${ }^{[18]}$.

同时, 我们可将总内能相应分解为可逆的部分 $e^{r}$ (称作自由内能)与不可逆的部分 $\psi($ (耗散能), 即 $e=e^{\mathrm{r}}+\psi$.

定义导热强度 $\mathbf{X}_{q}, \alpha$ 物质的扩散强度 $\mathbf{X}_{\alpha}^{\mu}$ 以及可逆 熵流 $\mathbf{J}_{q}$ 、可逆熵 $\eta^{\mathrm{r}}$ 为

$$
\begin{aligned}
& \mathbf{X}_{q}=-\nabla T, \mathbf{X}_{\alpha}^{\mu}=-\nabla \mu_{\alpha}, \\
& \mathbf{J}_{q}=\mathbf{j}_{q} / T, \\
& \dot{\eta}^{\mathrm{r}}=-\nabla \cdot \mathbf{J}_{q} .
\end{aligned}
$$

不失一般性, 对于考虑化学反应及其他内变量作 用的情况，由式(16)和(17)可得相应的局域形式内能 分解:

$$
\begin{aligned}
\dot{e}^{\mathrm{r}}= & \boldsymbol{\sigma}: \dot{\boldsymbol{\varepsilon}}^{\mathrm{r}}+T \dot{\eta}^{\mathrm{r}}+\sum_{\alpha} \mu_{\alpha} \dot{c}_{\alpha} \\
& -\sum_{\gamma} A^{(\gamma)} \dot{\ell}^{(\gamma)}-\sum_{N} \Gamma_{N} \dot{\chi}_{N}, \\
\dot{\psi}= & \boldsymbol{\sigma}: \dot{\boldsymbol{\varepsilon}}^{\mathrm{i}}+\mathbf{J}_{q} \cdot \mathbf{X}_{q}+\sum_{\alpha} \mathbf{j}_{\alpha}^{\mathrm{m}} \cdot \mathbf{X}_{\alpha}^{\mu} \\
& +\sum_{\gamma} A^{(\gamma)} \dot{\ell}^{(\gamma)}+\sum_{N} \Gamma_{N} \dot{\chi}_{N},
\end{aligned}
$$

其中, $A^{(\gamma)}$ 为 $\ell^{(\gamma)}$ 对应的化学亲和势; $\chi_{N}$ 表示第 $N$ 个内变 量, 而 $\Gamma_{N}$ 为其对应的热力学力. 由式(20)显然有 $e^{\mathrm{r}}=e^{\mathrm{r}}\left(\boldsymbol{\varepsilon}^{\mathrm{r}}, \eta^{\mathrm{r}}, c_{\alpha}, \ell^{(\gamma)}, \chi_{N}\right)$, 且

$$
\begin{gathered}
\boldsymbol{\sigma}=\frac{\partial e^{\mathrm{r}}}{\partial \boldsymbol{\varepsilon}^{\mathrm{r}}}, \quad T=\frac{\partial e^{\mathrm{r}}}{\partial \eta^{\mathrm{r}}}, \quad \mu_{\alpha}=\frac{\partial e^{\mathrm{r}}}{\partial c_{\alpha}}, \\
A^{(\gamma)}=-\frac{\partial e^{\mathrm{r}}}{\partial \ell^{(\gamma)}}, \quad \Gamma_{N}=-\frac{\partial e^{\mathrm{r}}}{\partial \chi_{N}} .
\end{gathered}
$$

式(17), (20)与(21)即力-热-化多场耦合问题内能 分解的一般形式, 其中式(20)右端5项分别体现了可逆 变形、可逆摘、物质扩散浓度、化学反应进度、内变 量对自由内能的影响，而式(21)则反映了不可逆变 形、热传导、物质扩散、化学反应、内变量所引起的 耗散能. 式(20)与式(21)清楚地表明了化学反应和内变 量变化不影响总内能, 它们所产生的耗散能等于自由 内能的减少, 即耗散能由自由内能转化而来. 顺便指 出, 文献 $[2,18,22,25]$ 中的耗散表达式均为式(21)的 特例.

\section{4 自由能与本构律}

本节将从热力学第二定律出发, 通过引入 Helmholtz自由能 $h=e-T \eta$, 推导多场耦合热力学系统的Clausius-Duhem不等式. 并将证明基于上一节内能分解的 热力学表述与基于传统自由能的热力学表述的一 致性.

热力学第二定律可由下述积分形式表述:

$$
\frac{\mathrm{d}}{\mathrm{d} t} \int_{\Omega} \eta \mathrm{d} V \geq-\int_{\partial \Omega} \frac{\mathbf{j}_{q} \cdot \mathbf{n}}{T} \mathrm{~d} S,
$$

其中, $\eta$ 是单位体积的总摘, 称为总摘密度. 在小变形假 设下, 可将式(23)改写为如下局域形式:

$$
\Phi=T \dot{\eta}^{\mathrm{i}}=T \dot{\eta}+\nabla \cdot \mathbf{j}_{q}+\mathbf{J}_{q} \cdot \mathbf{X}_{q} \geq 0,
$$

式中, $\Phi$ 即为由熵不等式定义的耗散功率, 不可逆熵 $\eta^{\mathrm{i}}=\eta-\eta^{\mathrm{r}}$.

由Helmholtz自由能的定义, 可以得到 $T \dot{\eta}=\dot{e}-$ $\dot{T} \eta-\dot{h}$, 结合能量守恒方程式(16), 可得Clausius-Duhem不等式:

$$
\begin{aligned}
\Phi= & T \dot{\eta}^{\mathrm{i}}=\boldsymbol{\sigma}: \dot{\boldsymbol{\varepsilon}}^{\mathrm{r}}+\boldsymbol{\sigma}: \dot{\boldsymbol{\varepsilon}}^{\mathrm{i}}+\mathbf{J}_{q} \cdot \mathbf{X}_{q}+\sum_{\alpha} \mu_{\alpha} \dot{c}_{\alpha} \\
& +\sum_{\alpha} \mathbf{j}_{\alpha}^{\mathrm{m}} \cdot \mathbf{X}_{\alpha}^{\mu}-\eta \dot{T}-\dot{h} \geq 0 .
\end{aligned}
$$

假设 $h=h\left(\boldsymbol{\varepsilon}^{\mathrm{r}}, T, c_{\alpha}, \ell^{(\gamma)}, \chi_{N}\right)$, 则上式可写成

$$
\begin{aligned}
& \left(\boldsymbol{\sigma}-\frac{\partial h}{\partial \mathbf{\varepsilon}^{\mathrm{r}}}\right): \dot{\boldsymbol{\varepsilon}}^{\mathrm{r}}-\left(\eta+\frac{\partial h}{\partial T}\right) \dot{T} \\
& \quad+\sum_{\alpha}\left(\mu_{\alpha}-\frac{\partial h}{\partial c_{\alpha}}\right) \dot{c}_{\alpha}+\boldsymbol{\sigma}: \dot{\boldsymbol{\varepsilon}}^{\mathrm{i}}+\mathbf{J}_{q} \cdot \mathbf{X}_{q} \\
& \quad+\sum_{\alpha} \mathbf{j}_{\alpha}^{\mathrm{m}} \cdot \mathbf{X}_{\alpha}^{\mu}+\sum_{\gamma} \widetilde{A}^{(\gamma)} \dot{\ell}^{(\gamma)}+\sum_{N} \widetilde{\Gamma}_{N} \dot{\chi}_{N} \\
& \quad \geq 0
\end{aligned}
$$


式中,

$$
\widetilde{A}^{(\gamma)}=-\frac{\partial h}{\partial \ell(\gamma)}, \quad \widetilde{\Gamma}_{N}=-\frac{\partial h}{\partial \chi_{N}} .
$$

式(26)左端每一项都代表一种独立的耗散机制 ${ }^{[22]}$, 进一步假设 $\boldsymbol{\sigma}, \mu_{\alpha}, \eta$ 不依赖于 $\dot{\boldsymbol{\varepsilon}}^{\mathrm{r}}, \dot{c}_{\alpha}, \dot{T}$, 则有

$$
\boldsymbol{\sigma}=\frac{\partial h}{\partial \boldsymbol{\varepsilon}^{\mathrm{r}}}, \quad \eta=-\frac{\partial h}{\partial T}, \quad \mu_{\alpha}=\frac{\partial h}{\partial c_{\alpha}} .
$$

于是耗散不等式简化为

$$
\begin{aligned}
\Phi= & T \dot{\eta}^{\mathrm{i}}=\boldsymbol{\sigma}: \dot{\boldsymbol{\varepsilon}}^{\mathrm{i}}+\mathbf{J}_{q} \cdot \mathbf{X}_{q} \\
& +\sum_{\alpha} \mathbf{j}_{\alpha}^{\mathrm{m}} \cdot \mathbf{X}_{\alpha}^{\mu}+\sum_{\gamma} \widetilde{A}^{(\gamma)} \dot{\ell}^{(\gamma)} \\
& +\sum_{N} \widetilde{\Gamma}_{N} \dot{\chi}_{N}
\end{aligned}
$$$$
\geq 0 \text {. }
$$

同理，对于其他的自由能形式也可类似得到相应 的本构方程及耗散不等式. 例如, 定义机械Gibbs自由 能 $g^{\mathrm{t}}=e-T \eta-\boldsymbol{\sigma}: \boldsymbol{\varepsilon}^{\mathrm{r}}$ 且 $g^{\mathrm{t}}=g^{\mathrm{t}}\left(\boldsymbol{\sigma}, T, c_{\alpha}, \ell^{(\gamma)}, \chi_{N}\right)$, 则相应 的第一类本构方程和耗散不等式分别为

$$
\begin{aligned}
\boldsymbol{\varepsilon}^{\mathrm{r}}= & -\frac{\partial g^{\mathrm{t}}}{\partial \boldsymbol{\sigma}}, \quad \eta=-\frac{\partial g^{\mathrm{t}}}{\partial T}, \quad \mu_{\alpha}=\frac{\partial g^{\mathrm{t}}}{\partial c_{\alpha}}, \\
\widetilde{\widetilde{A}}^{(\gamma)} & =-\frac{\partial g^{\mathrm{t}}}{\partial \ell^{(\gamma)}}, \quad \widetilde{\Gamma}_{N}=-\frac{\partial g^{\mathrm{t}}}{\partial \chi_{N}}, \\
\Phi= & T \dot{\eta}^{\mathrm{i}}=\boldsymbol{\sigma}: \dot{\boldsymbol{\varepsilon}}^{\mathrm{i}}+\mathbf{J}_{q} \cdot \mathbf{X}_{q} \\
& +\sum_{\alpha} \mathbf{j}_{\alpha}^{\mathrm{m}} \cdot \mathbf{X}_{\alpha}^{\mu}+\sum_{\gamma} \widetilde{\widetilde{A}}^{(\gamma)} \dot{\ell}^{(\gamma)} \\
& +\sum_{N} \widetilde{\widetilde{N}}_{N} \dot{\chi}_{N} \geq 0 .
\end{aligned}
$$

显然, 耗散能 $\psi$ 满足 $\psi=\Phi$. 对比式(21), (29)和(31), 可得

$$
A^{(\gamma)}=\widetilde{A}^{(\gamma)}=\widetilde{A}^{(\gamma)}, \Gamma_{N}=\widetilde{I}_{N}={\widetilde{\Gamma_{N}}} .
$$

这样内能的变化率可以写成

$$
\begin{aligned}
\dot{e}= & \dot{e}^{\mathrm{r}}+\dot{\psi}=\boldsymbol{\sigma}: \dot{\boldsymbol{\varepsilon}}^{\mathrm{r}}+T \dot{\eta}^{\mathrm{r}} \\
& +\sum_{\alpha} \mu_{\alpha} \dot{c}_{\alpha}-\sum_{\gamma} A^{(\gamma)} \dot{\ell}^{(\gamma)} \\
& -\sum_{N} \Gamma_{N} \dot{\chi}_{N}+T \dot{\eta}^{\mathrm{i}} \\
= & \boldsymbol{\sigma}: \dot{\boldsymbol{\varepsilon}}^{\mathrm{r}}+T \dot{\eta}+\sum_{\alpha} \mu_{\alpha} \dot{c}_{\alpha} \\
& -\sum_{\gamma} A^{(\gamma)} \dot{\ell}^{(\gamma)}-\sum_{N} \Gamma_{N} \dot{\chi}_{N} .
\end{aligned}
$$

因此 $e\left(\varepsilon^{\mathrm{r}}, \eta, c_{\alpha}, \ell^{(\gamma)}, \chi_{N}\right)$, 且有 $\boldsymbol{\sigma}=\frac{\partial e}{\partial \boldsymbol{\varepsilon}^{\mathrm{r}}}, \quad T=\frac{\partial e}{\partial \eta}, \quad \mu_{\alpha}=\frac{\partial e}{\partial c_{\alpha}}$,

$A^{(\gamma)}=-\frac{\partial e}{\partial \ell^{(\gamma)}}, \quad \Gamma_{N}=-\frac{\partial e}{\partial \chi_{N}}$.

式(22), (27)和(28), (30), (34)分别是用不同能量函数表 示的第一类本构方程.

\section{5 耗散能与演化方程}

根据热力学第二定律的约束, 容许的热力学状态 必须使得热力学流 $\mathbf{J}=\left\{\dot{\boldsymbol{\varepsilon}}^{\mathrm{i}}, \mathbf{J}_{q}, \mathbf{j}_{\alpha}^{\mathrm{m}}, \dot{\ell}^{(\gamma)}, \dot{\chi}_{N}\right\}^{\mathrm{T}}$ 及与其功率 共轭的热力学力 $\mathbf{X}=\left\{\boldsymbol{\sigma}, \mathbf{X}_{q}, \mathbf{X}_{\alpha}^{\mu}, A^{(\gamma)}, \Gamma_{N}\right\}^{\mathrm{T}}$ 满足耗散不 等式(29), 即

$\Phi=\dot{\psi}=\mathbf{X}^{\mathrm{T}} \mathbf{J}=\sum_{i} X_{i} J_{i} \geq 0$,

其中, $J_{i}$ 和 $X_{i}$ 分别表示 $\mathbf{J}$ 和 $\mathbf{X}$ 的分量.

根据不可逆热力学的线性唯象律 ${ }^{[23,26,27]}$, 不可逆 过程的演化可由如下耦合演化方程描述:

$J_{i}=\sum_{j} K_{i j} X_{j}$ 或 $X_{i}=\sum_{j} K_{i j}^{-1} J_{j}$,

其中, 耦合系数 $K_{i j}^{-1}$ 与 $K_{i j}$ 均为常数, 且对于耦合系数 $K_{i j}$ $\left(K_{i j}^{-1}\right)$, 还需满足热力学第二定律的正定性要求 ${ }^{[23]}$ 、基 于空间对称性的Curie 原理 ${ }^{[14]}$ 和基于时间对称性的Onsager-Casimir倒易关系 ${ }^{[26,27]}$ 等约束.

式(36)即为描述热力学系统中流与力的线性唯象 律, 称为第二类本构或演化方程. 需要说明的是, 热传 导的Fourier定律、物质扩散的Fick定律、热与扩散耦 合作用的Soret-DuFour效应、流体力学的NewtonStokes定律以及化学反应和内变量的线性演化方程, 都是其特例 ${ }^{[22,28]}$.

\section{6 力-热-化完全耦合的多场理论框架及等效 积分弱形式}

为了构造数值计算方法, 需要在多场耦合问题控 制方程的基础上建立对应的等效积分弱形式. 为此, 考虑上述内能的分解则可得如式(22)的第一类本构方 程. 此时, 同样可以得到耗散式不等式: 


$$
\begin{aligned}
\dot{\psi}= & T \dot{\eta}^{\mathrm{i}}=\boldsymbol{\sigma}: \dot{\boldsymbol{\varepsilon}}^{\mathrm{i}}+\mathbf{J}_{q} \cdot \mathbf{X}_{q} \\
& +\sum_{\alpha} \mathbf{j}_{\alpha}^{\mathrm{m}} \cdot \mathbf{X}_{\alpha}^{\mu}+\sum_{\gamma} A^{(\gamma)} \dot{\ell}^{(\gamma)} \\
& +\sum_{N} \Gamma_{N} \dot{\chi}_{N} \geq 0,
\end{aligned}
$$

以及形同式(36)的演化方程, 即第二类本构方程.

用熵表示的能量守恒方程(称为摘平衡方程)可写 为 ${ }^{[22,25]}$

$$
\begin{aligned}
T \dot{\eta}= & -\nabla \cdot \mathbf{j}_{q}+\boldsymbol{\sigma}: \dot{\boldsymbol{\varepsilon}}^{\mathrm{i}}+\sum_{\alpha} \mathbf{j}_{\alpha}^{\mathrm{m}} \cdot \mathbf{X}_{\alpha}^{\mu} \\
& +\sum_{\gamma} A^{(\gamma)} \dot{\ell}^{(\gamma)}+\sum_{N} \Gamma_{N} \dot{\chi}_{N} .
\end{aligned}
$$

事实上由 $\eta^{\mathrm{i}}=\eta-\eta^{\mathrm{r}}$ 和式(19), 可知在满足式(37)的前 提下, 式(19b)和(38)是等价的, 因此根据本文分解可逆 因素与不可逆因素的思想，可用反映可逆熵平衡的式 (19b)代替形式更复杂的熵平衡方程式(38). 于是, 多场 耦合问题中动量传递、热量传导、质量传输分别由动 量守恒方程式(11)、可逆熵平衡方程式(19b)和质量守 恒方程式(14)表示. 基于小变形与准静态假设, 上述三 类平衡方程具有如下可比拟的局域形式:

$\nabla \cdot \boldsymbol{\sigma}+\mathbf{f}=0, \quad \nabla \cdot \mathbf{J}_{q}+\dot{\eta}^{\mathrm{r}}=0, \quad \nabla \cdot \mathbf{j}_{\alpha}^{\mathrm{m}}+\dot{c}_{\alpha}=0$.
再考虑如下可比拟的边界条件.

第一类边界(Dirichlet boundary conditions)

$\mathbf{u}=\overline{\mathbf{u}}\left(\right.$ on $\left.\partial \Omega_{u}\right), T=\bar{T}\left(\right.$ on $\left.\partial \Omega_{T}\right)$,

$\mu_{\alpha}=\bar{\mu}_{\alpha}\left(\right.$ on $\left.\partial \Omega_{\mu_{\alpha}}\right)$.

第二类边界(Neumann boundary conditions)

$\boldsymbol{\sigma} \cdot \mathbf{n}=\overline{\mathbf{T}}\left(\right.$ on $\left.\partial \Omega_{u}\right), \mathbf{J}_{q} \cdot \mathbf{n}=\bar{J}_{q}\left(\right.$ on $\left.\partial \Omega_{\mathrm{J}_{q}}\right)$,

$\mathbf{j}_{\alpha}^{\mathrm{m}} \cdot \mathbf{n}=\overline{j_{\alpha}^{\mathrm{m}}}\left(\right.$ on $\left.\partial \Omega_{\mathbf{j}_{\alpha}^{\mathrm{m}}}\right)$,

其中,

$\partial \Omega=\partial \Omega_{u} \cup \partial \Omega_{\sigma}=\partial \Omega_{\mathbf{J}_{q}} \cup \partial \Omega_{T}=\partial \Omega_{\mathbf{j}_{\alpha}^{\mathrm{m}}} \cup \partial \Omega_{\mu}$,

$\partial \Omega_{u} \cap \partial \Omega_{\sigma}=\partial \Omega_{\mathbf{J}_{q}} \cap \partial \Omega_{T}=\partial \Omega_{\mathbf{j}_{\alpha}^{\mathrm{m}}} \cap \partial \Omega_{\mu}=\varnothing$.

则可得到由本构方程式(22)与(36)、平衡方程式(39)、 梯度关系式(18)、几何方程 $\boldsymbol{\varepsilon}=(\nabla \mathbf{u}+\mathbf{u} \nabla) / 2$ 和边界 条件式(40)和(41)构成的一个完整描述多场耦合问题 的理论框架，并记这些控制方程及相应边界条件构成 的封闭系统为边值问题 $\Lambda$.

进一步考虑上述边值问题 $\Lambda$ 中 16 类变量各自独立 变化, 则可构建如下等效积分弱形式:

$$
\begin{aligned}
& \delta \Pi=\int_{\Omega}\left(\frac{\partial e^{\mathrm{r}}}{\partial \boldsymbol{\varepsilon}^{\mathrm{r}}}-\boldsymbol{\sigma}\right): \delta \boldsymbol{\varepsilon}^{\mathrm{r}} \mathrm{d} V+\int_{\Omega}\left(\frac{\partial e^{\mathrm{r}}}{\partial c_{\alpha}}-\mu_{\alpha}\right) \delta c_{\alpha} \mathrm{d} V+\int_{\Omega}\left(\frac{\partial e^{\mathrm{r}}}{\partial \eta^{\mathrm{r}}}-T\right) \delta \eta^{\mathrm{r}} \mathrm{d} V \\
& +\int_{\Omega}\left(\frac{\partial e^{\mathrm{r}}}{\partial \ell^{(\gamma)}}+A^{(\gamma)}\right) \delta \ell^{(\gamma)} \mathrm{d} V+\int_{\Omega}\left(\frac{\partial e^{\mathrm{r}}}{\partial \chi_{N}}+\Gamma_{N}\right) \delta \chi_{N} \mathrm{~d} V \\
& +\int_{\Omega}\left[\dot{\boldsymbol{\varepsilon}}^{\mathrm{i}}-\left(K_{\sigma \sigma} \boldsymbol{\sigma}+\sum_{\alpha} K_{\sigma \mu(\alpha)} \mathbf{X}_{\alpha}^{\mu}+K_{\sigma T} \mathbf{X}_{q}+\sum_{\gamma} K_{\sigma A}^{(\gamma)} A^{(\gamma)}+\sum_{N} K_{\sigma \Gamma(N)} \Gamma_{N}\right)\right]: \delta \boldsymbol{\sigma} \mathrm{d} V \\
& +\int_{\Omega}\left[\mathbf{j}_{\alpha}^{\mathrm{m}}-\left(K_{\mu \sigma}^{(\alpha)} \boldsymbol{\sigma}+K_{\mu \mu}^{(\alpha)} \mathbf{X}_{\alpha}^{\mu}+K_{\mu T}^{(\alpha)} \mathbf{X}_{q}+\sum_{\gamma} K_{\mu A}^{(\alpha)(\gamma)} A^{(\gamma)}+\sum_{N} K_{\mu \Gamma(N)}^{(\alpha)} \Gamma_{N}\right)\right] \cdot \delta \mathbf{X}_{\alpha}^{\mu} \mathrm{d} V \\
& +\int_{\Omega}\left[\mathbf{J}_{q}^{T}-\left(K_{T \sigma} \boldsymbol{\sigma}+\sum_{\alpha} K_{T \mu(\alpha)} \mathbf{X}_{\alpha}^{\mu}+K_{T T} \mathbf{X}_{q}+\sum_{\gamma} K_{T A}^{(\gamma)} A^{(\gamma)}+\sum_{N} K_{T \Gamma(N)} T_{N}\right)\right] \cdot \delta \mathbf{X}_{q}^{T} \mathrm{~d} V \\
& +\int_{\Omega}\left[\dot{\ell}^{(\gamma)}-\left(K_{A \sigma}^{(\gamma)} \mathbf{\sigma}+\sum_{\alpha} K_{A \mu(\alpha)}^{(\gamma)} \mathbf{X}_{\alpha}^{\mu}+K_{A T}^{(\gamma)} \mathbf{X}_{q}+K_{A A}^{(\gamma)} A^{(\gamma)}+\sum_{N} K_{A \Gamma(N)}^{(\gamma)} \Gamma_{N}\right)\right] \delta A^{(\gamma)} \mathrm{d} V \\
& +\int_{\Omega}\left[\dot{\chi}_{N}-\left(K_{\Gamma \sigma}^{(N)} \boldsymbol{\sigma}+\sum_{\alpha} K_{\Gamma \mu(\alpha)}^{(N)} \mathbf{X}_{\alpha}^{\mu}+K_{I T}^{(N)} \mathbf{X}_{q}+\sum_{\gamma} K_{\Gamma A}^{(N)(\gamma)} A^{(\gamma)}+K_{I T}^{(N)} \Gamma_{N}\right)\right] \delta \Gamma_{N} \mathrm{~d} V \\
& +\int_{\Omega}\left[\frac{1}{2}(\nabla \mathbf{u}+\mathbf{u} \nabla)-\boldsymbol{\varepsilon}\right]: \delta \boldsymbol{\sigma} \mathrm{d} V+\int_{\Omega}\left(\mathbf{X}_{\alpha}^{\mu}+\nabla \mu_{\alpha}\right) \cdot \delta \mathbf{j}_{\alpha}^{\mathrm{m}} \mathrm{d} V+\int_{\Omega}\left(\mathbf{X}_{q}+\nabla T\right) \cdot \delta \mathbf{J}_{q} \mathrm{~d} V \\
& +\int_{\Omega} \boldsymbol{\sigma}: \delta(\nabla \mathbf{u}) \mathrm{d} V-\int_{\Omega} \dot{c}_{\alpha} \delta \mu_{\alpha} \mathrm{d} V+\int_{\Omega} \mathbf{j}_{\alpha}^{\mathrm{m}} \cdot \delta\left(\nabla \mu_{\alpha}\right) \mathrm{d} V-\int_{\Omega} \dot{\eta}^{\mathrm{r}} \delta T \mathrm{~d} V+\int_{\Omega} \mathbf{J}_{q}^{T} \cdot \delta(\nabla T) \mathrm{d} V \\
& -\int_{\Omega} \mathbf{f} \cdot \delta \mathbf{u d} V-\int_{\partial \Omega_{\sigma}} \overline{\mathbf{T}} \cdot \delta \mathbf{u d} S-\int_{\partial \Omega_{\mathrm{j}_{\alpha}}^{\mathrm{m}}} \bar{J}_{\alpha}^{\mathrm{m}} \delta \mu_{\alpha} \mathrm{d} S-\int_{\partial \Omega_{\mathrm{J}_{q}}} \bar{J}_{q}^{T} \delta T \mathrm{~d} S \\
& +\int_{\partial \Omega_{u}}(\overline{\mathbf{u}}-\mathbf{u}) \cdot \delta \boldsymbol{\sigma} \cdot \mathbf{n} \mathrm{d} S+\int_{\partial \Omega_{T}}(\bar{T}-T) \delta \mathbf{J}_{q} \cdot \mathbf{n} \mathrm{d} S+\int_{\partial \Omega_{\mu}}\left(\bar{\mu}_{\alpha}-\mu_{\alpha}\right) \delta \mathbf{j}_{\alpha}^{\mathrm{m}} \cdot \mathbf{n} \mathrm{d} S,
\end{aligned}
$$


其中演化方程系数阵 $\mathbf{K}$ 的各元素需满足上一节所述对 耦合系数 $K_{i j}$ 的各类约束.

显然, 由 $\delta \boldsymbol{\varepsilon}^{\mathrm{r}}, \delta \boldsymbol{\sigma}, \delta \boldsymbol{\varepsilon}^{\mathrm{i}}, \delta \mathbf{u}, \delta c_{\alpha}, \delta \mu_{\alpha}, \delta \mathbf{X}_{\alpha}^{\mu}, \delta \mathbf{j}_{\alpha}^{\mathrm{m}}, \delta \eta^{\mathrm{r}}, \delta T$, $\delta \mathbf{X}_{q}, \delta \mathbf{J}_{q}, \delta \ell^{(\gamma)}, \delta A^{(\gamma)}, \delta \chi_{N}, \delta \Gamma_{N}$ 的任意性易知, 泛函 $\Pi$ 的驻 值问题与前述边值问题完全等价，即式(42)为边值问 题 $\Lambda$ 的等效积分弱形式.

需要着重强调的是, 本节所得的式(42), 可称为力热-化多场耦合边值问题的一般等效积分弱形式. 通过 引入恰当的约束作为先决条件，可以得到不同形式的 退化等效积分弱形式. 例如，由本构方程式(22)与 (36)、几何方程与梯度关系式(18)以及边界条件式(40) 作为先决条件，则退化后的式(42)等价于平衡方程式 (39)和边界条件式(41)构成的边值问题，与文献中的多 场耦合拟变分原理等价 ${ }^{[18,20,29 \sim 31]}$.

\section{7 数值算例}

如图1所示, 考虑一个底部受刚性基底约束, 其他 部分自由的化学活性薄板, 将其浸入能扩散进入薄板 并发生化学反应的溶液中. 在各向同性假设下，该板 由于质量扩散和化学反应的影响将沿着厚度方向逐步 变形. 本节假设薄板满足线性的本构关系, 于是这样一 个扩散-反应-变形耦合问题可以由上述理论框架描述. 为了聚焦于当前问题以及方便与已有理论进行比较,
假设 $\chi_{N}=0$ 且 $A^{(\gamma)}=-\sum_{\alpha} v_{\alpha}^{(\gamma)} \mu_{\alpha}{ }^{[18,20,29 \sim 31]}$.

线性本构律假设下扩散-反应-变形耦合问题的自 由内能密度可写为

$$
\begin{aligned}
e^{\mathrm{r}}\left(\boldsymbol{\varepsilon}, c_{\alpha}, \ell^{(\gamma)}\right)= & e_{0}^{\mathrm{r}}+\boldsymbol{\sigma}_{0}: \boldsymbol{\varepsilon}+\sum_{\alpha} \mu_{\alpha}^{0} c_{\alpha} \\
& -\sum_{\gamma} A_{0}^{(\gamma)} \ell^{(\gamma)}+\frac{1}{2} \boldsymbol{\varepsilon}: \mathbf{C}: \boldsymbol{\varepsilon} \\
& +\sum_{\alpha} \mathbf{R}_{\alpha}: \boldsymbol{\varepsilon} c_{\alpha} \\
& -\sum_{\gamma} \boldsymbol{\beta}^{(\gamma)}: \mathbf{C}: \boldsymbol{\varepsilon} \ell^{(\gamma)} \\
& -\sum_{\gamma} \sum_{\alpha} \varsigma_{\alpha}^{(\gamma)} \ell^{(\gamma)} c_{\alpha} \\
& +\frac{1}{2} \sum_{\alpha} \mathcal{N}_{(\alpha)} c_{\alpha} c_{\alpha}-\frac{1}{2} \sum_{\gamma} a_{(\gamma)} \ell^{(\gamma)} \ell^{(\gamma)},
\end{aligned}
$$

其中, $\mathbf{C}$ 为对称的四阶刚度张量, $\mathbf{R}_{\alpha}, \boldsymbol{\beta}^{(\gamma)}, \varsigma_{\alpha}^{(\gamma)}$ 分别为扩 散-变形耦合张量、反应-变形耦合张量和反应-扩散耦 合系数, $\mathcal{N}_{(\alpha)}$ 为扩散场的本构系数, 而 $a_{(\gamma)}$ 为反应场的 本构系数. 此外, 我们不考虑塑性的影响, 所以 $\boldsymbol{\varepsilon}^{\mathrm{r}}=\boldsymbol{\varepsilon}$. 并 且由Curie原理, 阶数不同的 $\mathbf{X}_{\alpha}^{\mu}, A^{(\gamma)}$ 之间无耦合, 即系 数阵 $\mathbf{K}$ 中仅扩散演化参数 $K_{\mu \mu}^{(\alpha)}$ 和反应演化参数 $K_{A A}^{(\gamma)}$ 非零.

另需注意的是, 初始时刻 $t_{0}$ 时, 可以假设初始自由 能、化学势和亲和势为零, 同时薄板应力为自由状态, 即 $e_{0}^{\mathrm{r}}=0, \mu_{\alpha}^{0}=0, A_{0}^{(\gamma)}=0, \boldsymbol{\sigma}_{0}=\mathbf{0}$.

于是, 可以写出相应的等效积分弱形式:

$$
\begin{aligned}
\delta \Pi= & \int_{\Omega}(\mathbf{C}: \boldsymbol{\varepsilon}+R \mathbf{I} c-3 K \beta \ell-\boldsymbol{\sigma}): \delta \boldsymbol{\varepsilon} \mathrm{d} V+\int_{\Omega}(\mathcal{N} c+R \mathbf{I}: \boldsymbol{\varepsilon}-\varsigma \ell-\mu) \delta c \mathrm{~d} V \\
& +\int_{\Omega}(A-3 K \beta \mathbf{I}: \boldsymbol{\varepsilon}-\varsigma c-a \ell) \delta \ell \mathrm{d} V+\int_{\Omega}\left[\mathbf{j}^{\mathrm{m}}-K_{\mu \mu} \mathbf{X}^{\mu}\right] \cdot \delta \mathbf{X}^{\mu} \mathrm{d} V \\
& +\int_{\Omega}\left[\dot{\ell}-K_{A A} A\right] \delta A \mathrm{~d} V+\int_{\Omega}\left[\frac{1}{2}(\nabla \mathbf{u}+\mathbf{u} \nabla)-\boldsymbol{\varepsilon}\right]: \delta \boldsymbol{\sigma} \mathrm{d} V \\
& +\int_{\Omega}\left(\mathbf{X}^{\mu}+\nabla \mu\right) \cdot \delta \mathbf{j}^{\mathrm{m}} \mathrm{d} V+\int_{\Omega} \boldsymbol{\sigma}: \delta(\nabla \mathbf{u}) \mathrm{d} V-\int_{\Omega} \mathbf{f} \cdot \delta \mathbf{u} \mathrm{d} V \\
& -\int_{\Omega} \dot{c} \delta \mu \mathrm{d} V+\int_{\Omega} \mathbf{j}^{\mathrm{m}} \cdot \delta(\nabla \mu) \mathrm{d} V-\int_{\partial \Omega_{\sigma}} \overline{\mathbf{T}} \cdot \delta \mathbf{u d} S-\int_{\partial \Omega_{\mathbf{j}^{\mathrm{m}}}} \bar{J}^{\mathrm{m}} \delta \mu \mathrm{d} S \\
& +\int_{\partial \Omega_{u}}(\overline{\mathbf{u}}-\mathbf{u}) \cdot \delta \boldsymbol{\sigma} \cdot \mathbf{n} \mathrm{d} S+\int_{\partial \Omega_{\mu}}(\bar{\mu}-\mu) \delta \mathbf{j}^{\mathrm{m}} \cdot \mathbf{n} \mathrm{d} S .
\end{aligned}
$$

由于仅考虑一种物质扩散进出薄板且与之发生化 学反应，本节下标 $\alpha$ 及均可以省略. 在各向同性假设 下, 刚度系数 $\mathbf{C}$ 可以由两个独立的参数, 即体积模量 $K$ 和剪切模量 $G$ 表示, 其余二阶参数均退化为单值对角 阵常数. 本节算例忽略了体积力 $\mathbf{f}$ 的影响, 所取的材料

\section{参数如表 1 所示 ${ }^{[22]}$.}

利用COMSOL Multi-physics ${ }^{\circledR} 5.3$ 中数学组件的偏 微分方程弱形式模块, 求解得到了上述问题的解答. 图2展示了在不同的扩散和反应动力学参数下, 薄板 厚度方向不同位置应变随时间的演化. 其中记扩散动 


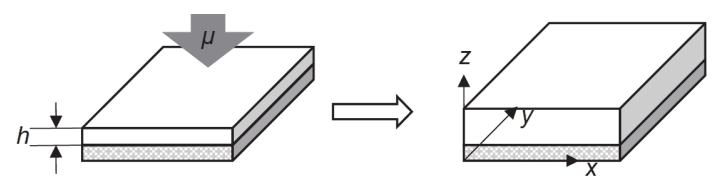

图 1 模型示意图

力学参数 $D=\mathcal{N} K^{\mu \mu}$ (即文献 $[17,22]$ 中的Fick定律扩散 系数), 以表征扩散过程的快慢; 记反应动力学参 数 $k_{\mathrm{knct}}=\varsigma K^{A A}$ (即文献[22]中的一阶反应动力学系数), 以衡量化学反应过程的快慢. 我们可进一步定义参 数 $\lambda_{t}=D / h^{2} k_{\mathrm{knct}}$, 用于衡量反应动力学特征时间与扩 散动力学特征时间之比.

图2(a)展示了一个典型的反应控制过程 $\left(\lambda_{t}>>1\right)$, 扩 散速率远高于反应速率, 扩散迅速达到平衡, 因此应变 很快趋于均匀分布，而后均匀应变的演化完全取决于 化学反应的进程; 图2(b)则对应一个典型的扩散控制 过程 $\left(\lambda_{t}<<1\right)$, 由于厚度方向化学势梯度的存在使得不 均匀应变不断随时间演化，足够长的时间才能使应变
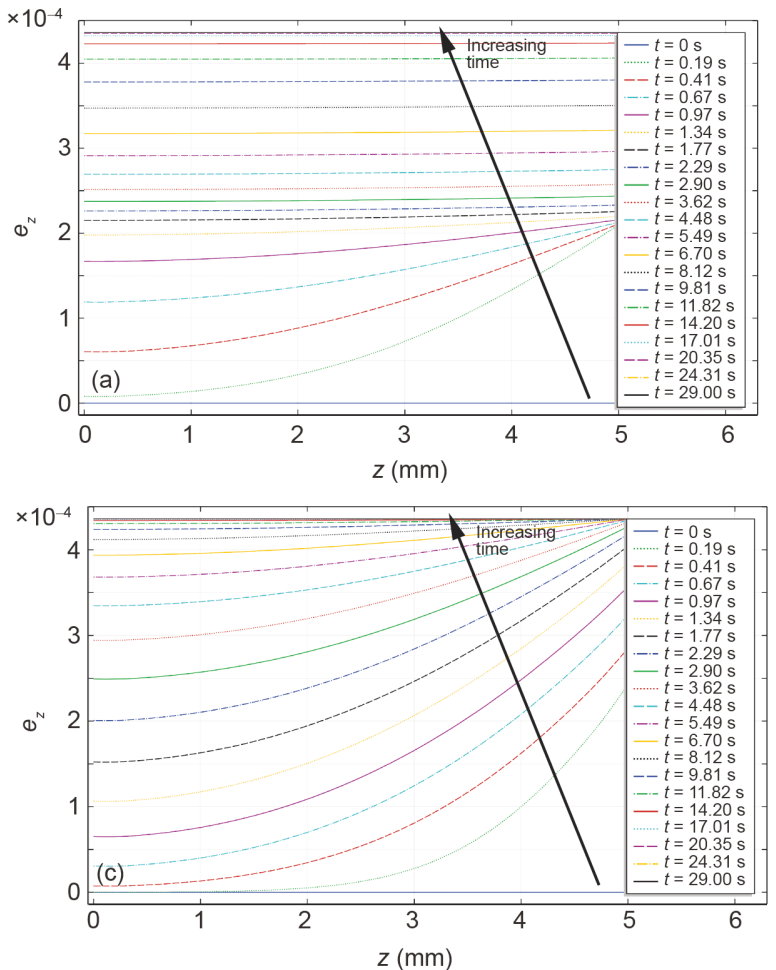

表 1 材料参数

\begin{tabular}{cc}
\hline 参数 & 数值 \\
\hline$K$ & $600 \times 10^{6} \mathrm{~Pa}$ \\
$G$ & $67 \times 10^{6} \mathrm{~Pa}$ \\
$R$ & $-4 \times 10^{3} \mathrm{~Pa} \mathrm{~m} / \mathrm{mol}$ \\
$\beta$ & $0.6 \times 10^{-5} \mathrm{~m}^{3} / \mathrm{mol}$ \\
$\mathcal{N}$ & $0.18 \mathrm{~J} \mathrm{~m}^{3} / \mathrm{mol}^{2}$ \\
$S$ & $1.2 \mathrm{~J} \mathrm{~m}^{3} / \mathrm{mol}^{2}$ \\
$a$ & $-1.1 \mathrm{~J} \mathrm{~m}^{3} / \mathrm{mol}^{2}$ \\
$K_{\mu \mu}$ & $4 \times 10^{-7} \sim 4 \times 10^{-5} \mathrm{~mol}^{2} /\left(\mathrm{J} \mathrm{m} \mathrm{s}^{2}\right)$ \\
$K_{A A}$ & $2.4 \times 10^{-3} \sim 2.4 \times 10^{-3} \mathrm{~mol}^{2} /\left(\mathrm{J} \mathrm{m}^{3} \mathrm{~s}\right)$ \\
$h$ & $5 \mathrm{~mm}$ \\
\hline
\end{tabular}

趋于均匀分布; 图2(c)与(d)体现了反应和扩散的速率 接近 $\left(\lambda_{t}=1\right)$ 时, 两种过程的耦合共同决定了应变的不均 匀分布. 因此, 参数 $\lambda_{t}$ 可以反映化学反应和扩散两种过 程的竞争情况.
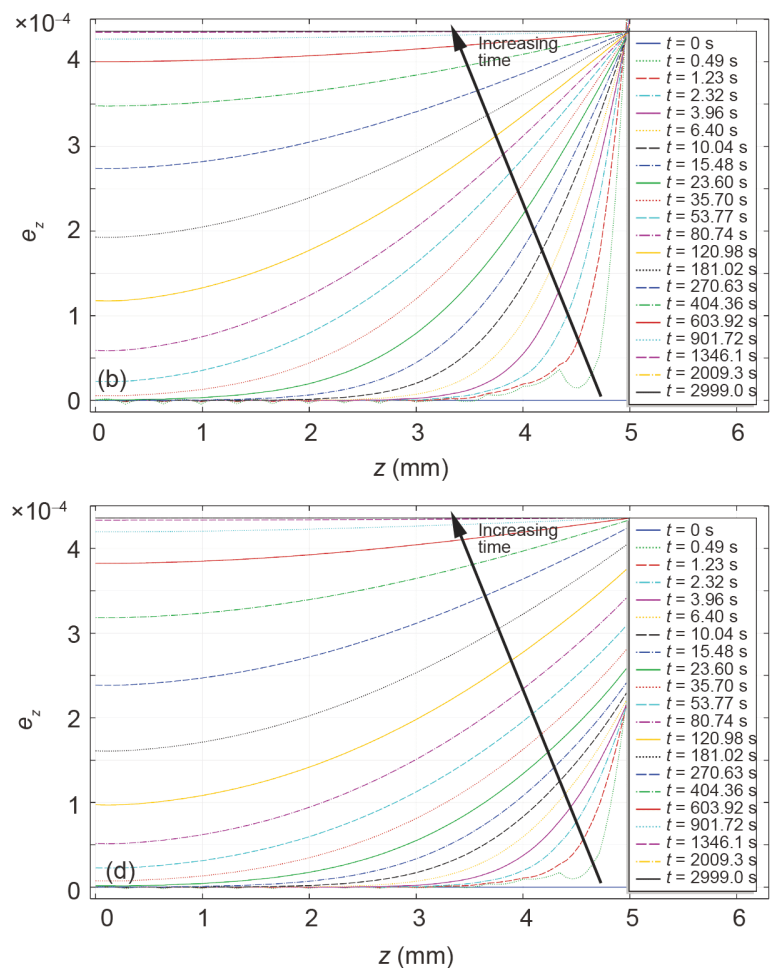

图 2 (网络版彩图)不同的扩散和反应动力学参数下，薄板纵向应变 $e_{z}$ 沿厚度方向的分布及其随时间的演化(箭头表示时间增 加的方向). (a) $D=0.72 \times 10^{-5} \mathrm{~m}^{2} / \mathrm{s}, k_{\mathrm{knc}}=2.88 \times 10^{-3} \mathrm{~s}^{-1}, \lambda_{t}=1 \times 10^{2}$; (b) $D=0.72 \times 10^{-7} \mathrm{~m}^{2} / \mathrm{s}, k_{\mathrm{knct}}=2.88 \times 10^{1} \mathrm{~s}^{-1}, \lambda_{t}=1 \times 10^{-4}$; (c) $D$ $=0.72 \times 10^{-5} \mathrm{~m}^{2} / \mathrm{s}, k_{\mathrm{knct}}=2.88 \times 10^{-1} \mathrm{~s}^{-1}, \lambda_{t}=1 ;$ (d) $D=0.72 \times 10^{-7} \mathrm{~m}^{2} / \mathrm{s}, k_{\mathrm{knct}}=2.88 \times 10^{-3} \mathrm{~s}^{-1}, \lambda_{t}=1$ 


\section{8 结论}

对于一般的开放系统, 本文给出了一个力-热-化 完全耦合的连续介质力学理论框架, 使得多场问题的 控制方程形式更加简洁、彼此之间的类比关系更加清 晰. 与传统理论不同的是, 本文将内能分解为可逆的自 由内能和不可逆的耗散能，这样的处理方式和所得到
的数学模型, 不仅可以清楚地区分出总内能中耗散的 能量, 也便于建立等效积分弱形式进行多场耦合问题 的数值模拟.

作为初步探索, 本文在小变形假设下构建了基于 内能分解的力-热-化多场耦合理论框架, 因此只适用 于小变形的力-热-化多场耦合问题. 对于大变形情况, 将在后续研究中加以考虑.

\section{参考文献}

1 Bekas D G, Tsirka K, Baltzis D, et al. Self-healing materials: A review of advances in materials, evaluation, characterization and monitoring techniques. Compos Part B-Eng, 2016, 87: 92-119

2 Chester S A, Anand L. A coupled theory of fluid permeation and large deformations for elastomeric materials. J Mech Phys Solids, 2010, 58: $1879-1906$

3 Brassart L, Suo Z. Reactive flow in large-deformation electrodes of lithium-ion batteries. Int J Appl Mech, 2012, 04: 1250023

4 Swaminathan N, Qu J M. A mechanical-electrochemical theory of defects in ionic solids. In: Bansal N P, Wereszczak A, Lara-Curzio E, eds. Advances in Solid Oxide Fuel Cells II: Ceramic Engineering and Science Proceedings, Volume 27. Hoboken: A John Wiley \& Sons, Inc., Publication, 2007. 125-136

5 Huyghe J, Janssen J D. Thermo-chemo-electro-mechanical formulation of saturated charged porous solids. Transp Porous Media, 1999, 34: 129141

6 Coussy O. Poromechanics. West Sussex: John Wiley \& Sons, 2004

7 Coussy O. Mechanics and Physics of Porous Solids. West Sussex: Wiley, 2010

8 Pan Y H, Zhong Z. The effect of hybridization on moisture absorption and mechanical degradation of natural fiber composites: An analytical approach. Compos Sci Tech, 2015, 110: 132-137

9 Pan Y H, Zhong Z. A nonlinear constitutive model of unidirectional natural fiber reinforced composites considering moisture absorption. J Mech Phys Solids, 2014, 69: 132-142

10 Peradzynski Z. Diffusion of calcium in biological tissues and accompanying mechano-chemical effects. Arch Mech, 2010, 62: 423-440

11 Yang C H, Zhou S, Shian S, et al. Organic liquid-crystal devices based on ionic conductors. Mater Horiz, 2017, 4: 1102-1109

12 Gibbs J W. The Scientific Papers of J. Willard Gibbs. London: Longmans, Green and Company, 1878. 184, 201, 215

13 Biot M A. General theory of three-dimensional consolidation. J Appl Phys, 1941, 12: 155-164

14 De Groot S R, Mazur P. Non-equilibrium Thermodynamics. New York: Courier Dover Publications, 2013

15 Chester S A, Srivastava V, Anand L. A thermo-mechanically coupled large-deformation theory for amorphous polymers across the glass transition temperature. In: Proceedings of the ASME International Mechanical Engineering Congress and Exposition (IMECE 2010 ), Vol 9. Vancouver, 2012. 669-675

16 Hong W, Zhao X, Zhou J, et al. A theory of coupled diffusion and large deformation in polymeric gels. J Mech Phys Solids, 2008, 56: 1779-1793

17 Yang Q S, Qin Q H, Ma L H, et al. A theoretical model and finite element formulation for coupled thermo-electro-chemo-mechanical media. Mech Mater, 2010, 42: 148-156

$18 \mathrm{Yu}$ P, Shen S. A fully coupled theory and variational principle for thermal-electrical-chemical-mechanical processes. J Appl Mech, 2014, 81: 111005

19 Haftbaradaran H, Qu J M. Two-dimensional chemo-elasticity under chemical equilibrium. Int J Solids Struct, 2015, 56-57: 126-135

20 Chen J, Wang H, Yu P, et al. A finite element implementation of a fully coupled mechanical-chemical theory. Int J Appl Mech, 2017, 09: 1750040

21 Sain T, Loeffel K, Chester S. A thermos-chemo-mechanically coupled constitutive model for curing of glassy polymers. J Mech Phys Solids, 2018, 116: 267-289

22 Zhang X L, Zhong Z. A coupled theory for chemically active and deformable solids with mass diffusion and heat conduction. J Mech Phys Solids, 2017, 107: 49-75 
23 Jou D, Casas-V Zquez J, Lebon G. Extended Irreversible Thermodynamics. New York: Springer, 2014

24 Truesdell C, Baierlein R. Rational Thermodynamics. New York: Springer-Verlag, 1984

25 Loeffel K, Anand L. A chemo-thermo-mechanically coupled theory for elastic-viscoplastic deformation, diffusion, and volumetric swelling due to a chemical reaction. Int J Plast, 2011, 27: 1409-1431

Coleman B D, Gurtin M E. Thermodynamics with internal state variables. J Chem Phys, 1967, 47: 597-613

Glansdorff P, Nicolis G, Prigogine I. The thermodynamic stability theory of non-equilibrium states. Proc Natl Acad Sci USA, 1974, 71: 197-199

Zhang X L, Zhong Z. A thermodynamic framework for thermo-chemo-elastic interactions in chemically active materials. Sci China-Phys Mech Astron, 2017, 60: 084611

29 Kuang Z B. Variational principles for generalized thermodiffusion theory in pyroelectricity. Acta Mech, 2010, 214: 275-289

30 Kuang Z B. Variational principles for generalized dynamical theory of thermopiezoelectricity. Acta Mech, 2009, 203: 1-11

$31 \mathrm{Hu}$ S L, Shen S P. Non-equilibrium thermodynamics and variational principles for fully coupled thermal-mechanical-chemical processes. Acta Mech, 2013, 224: 2895-2910

\title{
Continuum mechanics for thermo-chemo-mechanical coupling system based on decomposition of internal energy
}

\author{
ZHENG JiaHong $^{1}$, JIANG CongYing ${ }^{1} \&$ ZHONG Zheng ${ }^{2}$ \\ ${ }^{1}$ School of Aerospace Engineering and Applied Mechanics, Tongji University, Shanghai 200092, China; \\ ${ }^{2}$ School of Science, Harbin Institute of Technology, Shenzhen 518055, China
}

In this paper, based on the decomposition of internal energy into free internal energy and dissipative energy, some conservation laws of a thermodynamically open continuum system as well as related constitutive relations and evolution equations are derived when considering heat conduction, mass transfusion, chemical reactant and mechanical deformation. Upon establishing a weak form of corresponding governing differential equations and boundary conditions under above theoretical framework, a simple example is then numerically implemented to demonstrate the solution procedure.

continuum thermodynamics, multi-physics coupling, free energy, chemical reaction

doi: 10.1360/SST-2019-0053 